\title{
A Chemorheological Equation of State for the Stress Relaxation of Elastomers
}

\author{
Michael C. H. LEE* and Mitchel SHEN** \\ University of California, Berkeley, Berkeley, \\ California 94720, U.S.A.
}

(Received February 26, 1980)

\begin{abstract}
A chemorheological equation of state for the stress relaxation of elastomers has been derived. This equation of state provides a general relationship between stress ratio and aging time.

KEY WORDS Stress Ratio / Chemorheology / Aging Function / Primary Transient Network / Secondary Transient Network / Cross-Linking / Scission /
\end{abstract}

Elastomers under aging tests show the changes of the structures. These include the average molecular weight, the molecular weight distribution, the effective cross-linking density, and the transient networks, such as the entanglement density. Consequently, the properties of the elastomer system will also change. A useful method for determining the change in mechanical properties is the chemical-stress relaxation test. ${ }^{1-3}$ Detail information for the chemical-stress relaxation test can be found in the work by Kusano, Tamura, and Murakami. ${ }^{4}$ In their work, the plots between the logarithmic stress ratio and the aging time exhibit a family curves corresponding to various stretch ratios for a given elastomer. By defining the slope obtained from large aging time periods, they concluded that the apparant rate constant for an aging reaction is a function of the stretch ratio, the vibrational frequency, and the temperature.

From the chemorheological point of view, a chemorheological equation of state, obtained from a good mechanical constitutive equation and a good aging kinetics for the elastomer system, is required.

\section{THEORY}

Under uniaxial tensile deformation, a new

* Contact person's present address: General Motors Research Laboratories, Warren, Michigan 48090, U.S.A.

** During the preparation of this paper, we deeply regreted the loss of Professor Mitchel Shen. mechanical constitutive equation for gum elastomers has been derived by Lee and Williams ${ }^{5}$ in the form,

$$
\sigma_{\mathrm{E}}=G\left(2 C_{3} / G\right)^{\lambda^{-|b|}}\left(\lambda-\lambda^{-2}\right)
$$

where

$$
2 C_{3}=2 C_{2}+G
$$

Equation 1 is valid for elastomers which follow the Gaussian-chain dynamics during deformation. The term $2 C_{2}$ is the shear modulus due to the transient network, and $G$ is the shear modulus due to the chemical cross-links. The rate and temperature dependences of $G$ are given as

$$
G=G_{\mathrm{o}}(d)^{g} \exp (h / T)
$$

where $G_{0}$ is the zero shear modulus, $T$, the temperature, and $d$, the second invariant of deviatorial deformation rate tensor. The material constants $g$ and $h$ can be determined experimentally.

\section{Chemorheological Equation of State}

Based on eq 1, we now derive the chemorheological equation of state for gum elastomers. The shear modulus, $2 C_{2}$ is attributed to the transient networks such as the effective entanglements. Physically, $2 C_{2}$ contains two parts. They are

$$
2 C_{2}=2 C_{2 \mathrm{I}}+2 C_{2 \mathrm{II}}
$$

The first part, $2 C_{2 I}$ is the shear modulus due to the primary transient networks. The primary transient 
networks exist even without cross-linking reactions. The second part, $2 C_{2 \text { II }}$ is the shear modulus due to the secondary transient networks. The secondary transient networks are produced by chemical crosslinking reactions, and can be positive, null or negative depending on the chain morphology in which the cross-linking reaction occurs.

For example, if we consider the transient networks are composed of entanglements only, then an effective entanglement can be produced if the crosslinking reaction occurs at a proper site as shown in Figure 1. However, if the cross-linking reaction happens at the site of the entanglement which has already existed before the cross-linking reaction, then we have a negative secondary entanglement. Figure 2 illustrates the point we have just discussed. The secondary transient network is caused only by the cross-linking reaction. Consequently, the shear
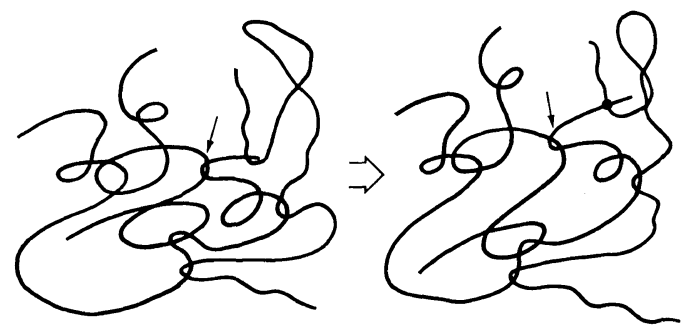

Figure 1. The evolution of a positive secondary entanglement in a cross-linking process. The dot indicates the cross-linking site, and the arrow indicates the entanglement site, which is an ineffective entanglement before the cross-linking reaction.
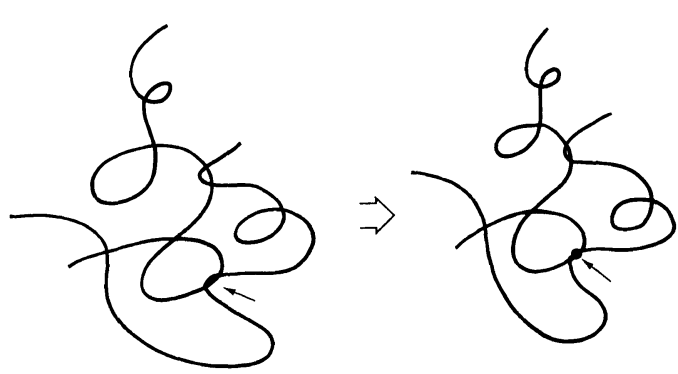

Figure 2. The evolution of a negative secondary entanglement in a cross-linking process. The arrow indicates an entanglement, and the dot indicates a crosslinking site. In this case the cross-linking reaction occurs at the entanglement site. modulus $2 C_{2 \text { II }}$ is only a function of the shear. modulus $G$, and $G$ is a function of the chemical cross-linking density. We accordingly define the relationship between $2 C_{2 \text { II }}$ and $G$ as

$$
2 C_{2 \mathrm{II}}=r(G)^{v}
$$

where $r$ and $v$ are two material constants which can be determined experimentally. The value of $v$ is always greater than zero. However, the parameter $r$ has the following properties:

$$
\begin{array}{ll}
r>0 \text { implies } & 2 C_{2 \mathrm{II}}>0 \\
r=0 \text { implies } & 2 C_{2 \mathrm{II}}=0 \\
r<0 \text { implies } & 2 C_{2 \mathrm{II}}<0
\end{array}
$$

Combining eq 3 and 4 leads to

$$
2 C_{2}=2 C_{2 \mathrm{I}}+r(G)^{v}
$$

The schematic plots of eq 5 are shown in Figure 3. The first systematical experiment for the transient network was investigated by Moore and Watson. ${ }^{6}$ Based on their experimental data for natural rubber, we obtain $2 C_{2 \mathrm{I}}=0.13 \mathrm{MNm}^{-2}, v=1$ and $r=0.205$ The results are shown in Figure 4.

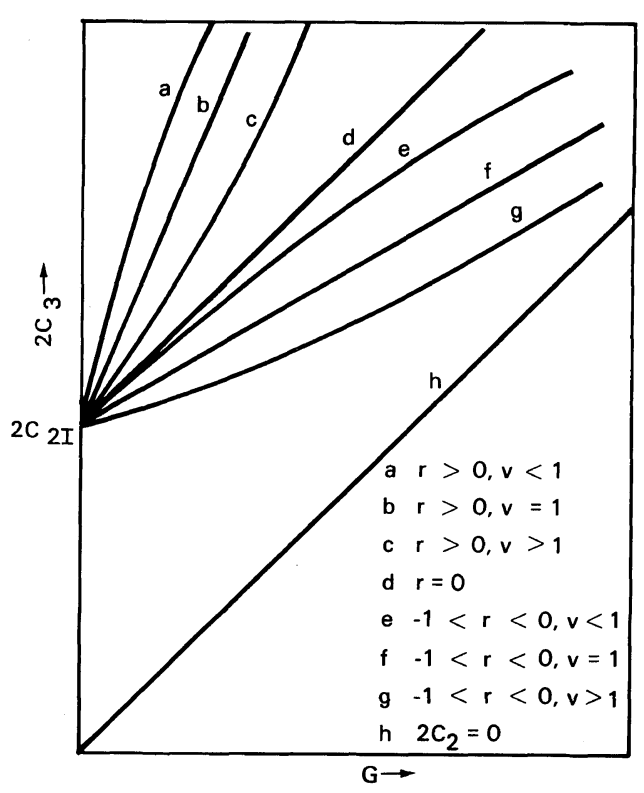

Figure 3. A schematic plot between the total shear modulus $2 C_{3}$ and the shear modulus due to permanent cross-links $G$. In this figure, eight different cases are plotted. 
Flory $^{7}$ has established a relationship between the shear modulus and the cross-linking density in the form,

$$
G(t)=N(t) R T
$$

Consequently, we obtain

$$
G(t) / G(0)=N(t) / N(0)
$$

In eq 7, $G(t)$ and $N(\mathrm{t})$ are the shear modulus and the cross-linking density at aging time $t$ respectively, and $G(0)$ and $N(0)$ are the initial shear modulus and the initial cross-linking density, respectively.

We now define the aging function for a chemorheological elastomer system as

$$
A(t)=N(t) / N(0)
$$

Under a constant stretch ratio $\lambda$, eq 1 leads to the stress ratio as

$$
\frac{\sigma_{\mathrm{E}}(t)}{\sigma_{\mathrm{E}}(0)}=\left[\frac{G(t)}{G(0)}\right]\left[\frac{2 C_{3}(t) G(0)}{2 C_{3}(0) G(t)}\right]^{\lambda-b}
$$

Finally, combining eq 8,5 , and 9 , we obtain
Equation 10 is the chemorheological equation of state for the stress-relaxation test of gum elastomer.

It is noteworthy that $2 C_{2 \mathrm{I}}$ in eq 10 is assumed to be time-independent on the basis that the primary transient network represents a steady-state value of network density even without the cross-linking reactions.

\section{Aging Function $A(t)$ and Chemical Reactions}

For a system with $i$ parallel reactions, the overall aging function can be expressed as

$$
A(t)=\sum A_{i}(t)
$$

where $A_{i}(t)$ is the aging function of the $i$ th reaction. For a system with $i$ sequential reactions, the total aging function can then be expressed as

$$
[1 / A(t)]=\Sigma\left[1 / A_{i}(t)\right]
$$

For example, if a system has a $m$ th-order chemical reaction, then the aging function can be expressed as

$$
A(t)=\left\{[G(0) / R T]^{1-m}(1-m) K t+1\right\}^{1 /(m-1)}
$$

$$
\frac{\sigma_{\mathrm{E}}(t)}{\sigma_{\mathrm{E}}(0)}=A(t)\left[\frac{1+r\{G(0)\}^{v-1}\{A(t)\}^{v-1}+A(t)^{-1}\left\{2 C_{2 \mathrm{I}} / G(0)\right\}}{1+r\{G(0)\}^{v-1}+2 C_{2 \mathrm{I}} / G(0)}\right]^{\lambda-b}
$$

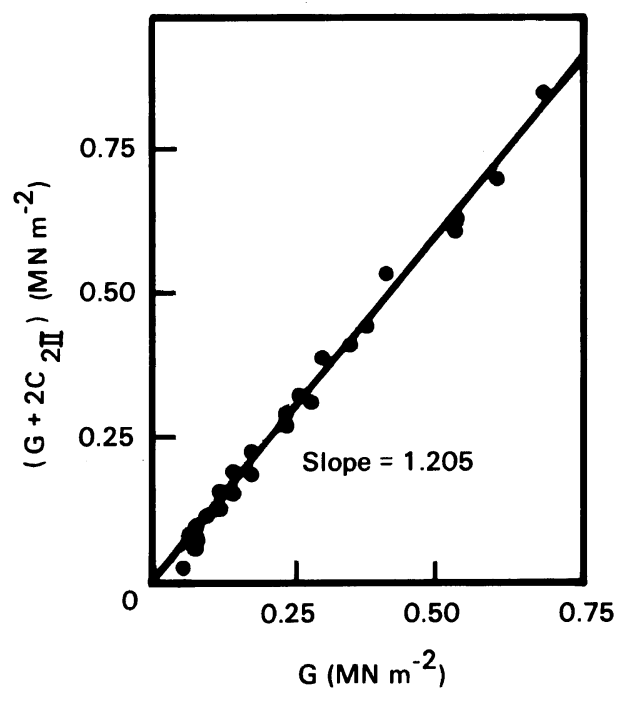

Figure 4. A plot between the secondary transient shear modulus $2 C_{2 \text { II }}$ and the shear modulus due to permanent cross-links $G$ for peroxide cured natural rubber. Data are taken from Moore and Watson (1965).

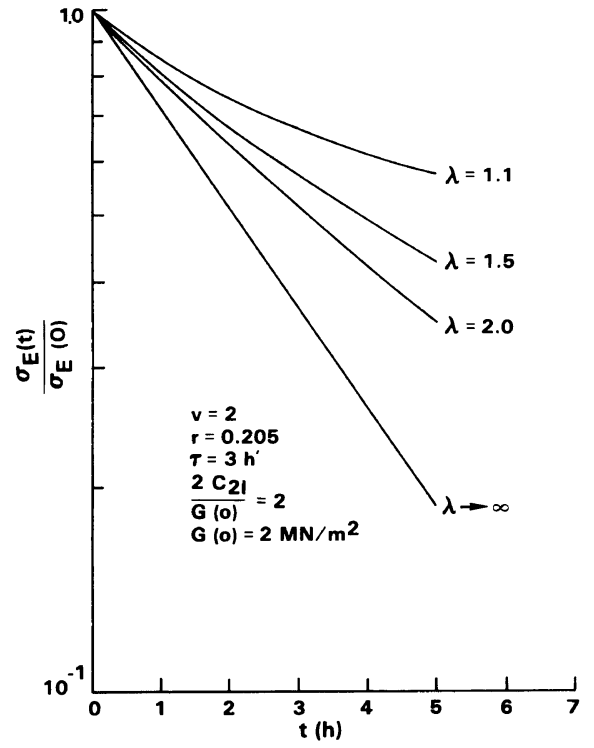

Figure 5. A theoretical prediction between the stress ratio and aging time under various stretch ratios of a chemical-stress relaxation test. 
It is important to note that in this case the aging function depends on the cross-linking density as well as on the apparent rate constant, $K$.

Consider a system with $n$ parallel first-order reactions, then the aging function can be expressed as

$$
A(t)=\sum \exp \left(-K_{i} t\right)
$$

where

\section{First-Order Chain Scission Reactions}

For a first-order chain scission reaction, the aging function $A(t)$ can be expressed as

$$
A(t)=\exp (-K t)=\exp (-t / \tau) ; \quad K>0
$$

It is noteworthy that $A(t)$ depends only on the apparent rate constant in this case. Combining eq 15 and 10 , we obtain

$$
\frac{\sigma_{\mathrm{E}}(t)}{\sigma_{\mathrm{E}}(0)}=\exp (-t / \tau)[W(t)]
$$

$$
W(t)=\left[\frac{1+r\{G(0)\}^{v-1} \exp \{-(v-1) t / \tau\}+\left\{2 C_{2 \mathrm{I}} / G(0)\right\} \exp (t / \tau)}{1+r\{G(0)\}^{v-1}+2 C_{2 \mathrm{I}} / G(0)}\right]^{\lambda-b}
$$

In eq 16 , the stress ratio not only depends on the rate and temperature through $G(0)$, but also on the stretch ratio. A theoretical prediction of eq 16 is shown in Figure 5. It is important to note that the chemical-stress relaxation curve depends on the stretch ratio, even though the apparent rate constant is constant.

If $2 C_{2}$ is zero, which implies null transient network in the system, eq 15 reduces to

$$
\frac{\sigma_{\mathrm{E}}(t)}{\sigma_{\mathrm{E}}(0)}=\exp (-t / \tau)
$$

Equation 17 has the same form that was suggested by Tobolsky. ${ }^{8}$

\section{REFERENCES}

1. A. V. Tobolsky, J. Appl. Phys., 27, 673 (1956); A. V.
Tobolsky, "Property and Structure of Polymers," Wiley, New York, N.Y., 1960, p 228.

2. A. V. Tobolsky and K. Murakami, J. Polym. Sci., 40, 443 (1959).

3. R. B. Beevers, J. Colloid Sci., 19, 40 (1964).

4. T. Kusano, S. Tamura, and K. Murakami, J. Polym. Sci., Polym. Symp., No. 46, 251 (1974).

5. M. C. H. Lee and M. C. Williams, presented at the Second U.S.-Japan Joint Rheology Meeting, Kona, Hawaii, April 8, 1979. To be published in J. Rheol.

6. C. G. Moore and W. F. Watson, J. Polym. Sci., 19, 237 (1956).

7. P. J. Flory, "Statistical Mechanics of Chain Molecules," Wiley (Interscience), New York, N.Y., 1969.

8. A. V. Tobolsky, J. Polym. Sci., B, 2, 823 (1964). 